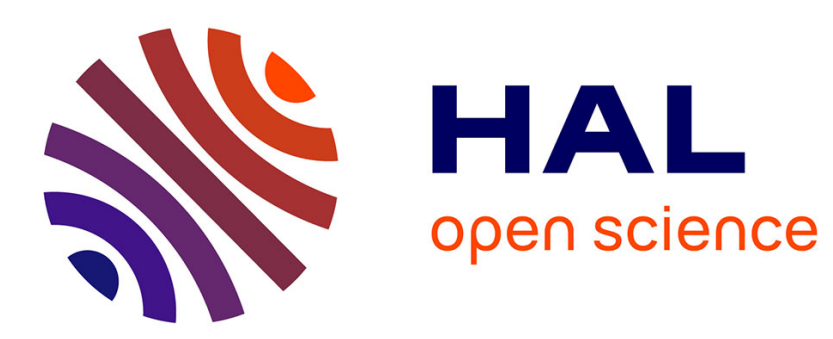

\title{
On constructing a logic for the notion of complete and immediate formal grounding
}

Francesca Poggiolesi

\section{To cite this version:}

Francesca Poggiolesi. On constructing a logic for the notion of complete and immediate formal grounding. Synthese, 2017, 10.1007/s11229-016-1265-z . halshs-01474697

\section{HAL Id: halshs-01474697 \\ https://shs.hal.science/halshs-01474697}

Submitted on 26 Jun 2018

HAL is a multi-disciplinary open access archive for the deposit and dissemination of scientific research documents, whether they are published or not. The documents may come from teaching and research institutions in France or abroad, or from public or private research centers.
L'archive ouverte pluridisciplinaire HAL, est destinée au dépôt et à la diffusion de documents scientifiques de niveau recherche, publiés ou non, émanant des établissements d'enseignement et de recherche français ou étrangers, des laboratoires publics ou privés. 


\title{
Francesca Poggiolesi \\ On constructing a logic for the notion of complete and immediate formal ground- ing
}

\begin{abstract}
In [14] we have introduced a rigorous definition of the notion of complete and immediate formal grounding; in the present paper our aim is to construct a logic for the notion of complete and immediate formal grounding based on that definition. Our logic will have the form of a calculus of natural deduction, will be proved to be sound and complete and will allow us to have fine-grained grounding principles.
\end{abstract}

\section{Introduction}

In the last ten years there has been a growing interest in the concept of grounding - when one truth holds because of others - with several different studies contributing to the development of our understanding of this concept (e.g. $[7,8,16,17])$. Grounding is a complex notion that can be classified in several ways. Most of the philosophical literature focuses on metaphysical grounding (e.g. see [2]), but there are also natural grounding, normative grounding and logical grounding (e.g. see [9]). In this paper, in line with the works of [5, 10, 18], we will restrict our attention to logical grounding, that we will refer to as formal grounding, for the sake of harmony with [14].

As concerns the notion of formal grounding, two other classifications can be introduced. The first concerns the distinction between immediate and mediate grounding. As Tatzel [20, p. 9] says "immediate grounding is essentially a oneone relation"; mediate grounding, on the other hand, can be introduced as the transitive closure of the relation of immediate grounding. This terminology has been firstly adopted by Bolzano [3, §203-219] and then independently used by Fine [9].

The second distinction of interest for us is that between complete and partial grounding. According to Bolzano (see [19] and [20, p.13]), the (multi)set of all, and only, those truths each of which contributes to ground the truth $C$ is a complete ground of $C$. On the other hand, each of the truths that composes the complete ground of $C$ is said to be a partial ground of $C$. Fine has a slightly different conception of the distinction between complete and partial ground, see [13] for arguments in support of the Bolzanian distinction.

In this paper, we will focus on the notion of complete and immediate formal grounding and provide a propositional logic for this notion. This logic can be thought of as the formal counterpart of the definition of the notion of complete 
and immediate formal grounding that we have introduced and conceptually defended in [14]. Thus the technical part of [14] will be briefly recalled here (the present paper can hence be thought of as technically self-contained), while for the philosophical considerations behind the definition of complete and immediate grounding the reader is referred to [14].

The paper is organized as follows. Since other logics of grounding have already been proposed recently (see $[4,5,9,10,18]$ ), we will use Section 2 to explain our main contributions and how our approach differs from others. In Section 3 we will recall the definition of complete and immediate formal grounding, which our logic of grounding aims to capture; in Section 4 we will introduce

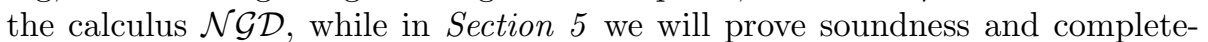
ness. In Section 6 we will discuss some technical and conceptual features of the

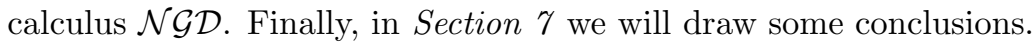

\section{Detailed comparison with other logics of ground- ing}

In this section we will outline the central contributions of our proposal, in particular with respect to the existing literature. We will first present those characteristics of our logic of grounding which are entirely new, before turning to more subtle points of comparison with previous approaches. Other important contributions of this work will be also commented on in Section 6 .

The first noteworthy characteristic of our logic for the notion of complete and immediate formal grounding lies in the rule for negation. Our logic is the first logic to have an unique rule for the negation connective. This rule validates grounding principles for negation analogous to those of $[5,9,18]$, but it also shows what unites and motivates these grounding principles. The rule indeed formalizes the following grounding-analysis of the negation connective. Consider a negative truth like "the ball is not cubic" and try to answer the question: what is the (complete and immediate) ground of this truth? The ground is "the ball is spherical". Indeed, if "the ball is spherical" is true, and "the ball is spherical" and "the ball is cubic" lead to a contradiction, then "the ball is spherical" is the ground of "the ball is not cubic." In more formal terms, the idea here is that the ground for the truth of a formula of the form $\neg A$ is a formula $B$ (satisfying a certain complexity constraint) such that $A$ and $B$ lead to a contradiction.

A second characteristic of our logic for the notion of complete and immediate formal grounding lies in the rules for the disjunction connective. ${ }^{1}$ These rules indeed contain a novel element called robust conditions that provide an original grounding-analysis of disjunction. In order to explain what robust conditions are in a brief way, consider the example of a disjunction like $A \vee B$, in the situation where the formula $A$ is true. In this case, $A$ is certainly a ground for $A \vee B$; but in order for $A$ to be the complete ground of $A \vee B$, it is necessary to

\footnotetext{
${ }^{1}$ Analogous considerations hold for the grounding principles governing negation of conjunction.
} 
specify that $B$ is false (i.e. that $B$ is not a ground for $A \vee B$ ); in other terms, it is the falsity of $B$ that ensures that, or is a (robust) condition for $A$ to be the complete ground of $A \vee B$. Thus, $A$ is the complete and immediate formal ground of $A \vee B$ under the robust condition that $B$ is false. ${ }^{2}$ The reader is refered to [14] for a detailed explanation and discussion of the idea of robust conditions in a grounding framework. Robust conditions will be denoted by square brackets and will be introduced in Definition 3.7.

A third notable characteristic of our work relates to an important debate in the current literature on how to best express talk about grounding. Standardly, logics of grounding restrict themselves to considering grounding merely via a symbol of a formal language, be it a predicate or a sentential operator. Our approach stands apart, and will be based on a relationship between grounding and a certain sort of proofs, called proofs-why. Our starting point is indeed the famous distinction, which dates back at least as far as Aristotle [1, I.13] and was adopted by Bolzano [3, §525], between two types of proofs: proofs that show that a conclusion is true (proofs-that), and proofs that show why a conclusion is true (proofs-why). Proofs-that are composed by inferential steps: in each of these steps the truth of the premisses allows one to infer that the conclusion is true as well. Proofs-why, on the other hand, are explanatory proofs: they establish not just the truth of the conclusion but reveal the premises to be the reasons why the conclusion is true. They are thus intimately related to the question of grounding: in showing why the conclusion is true, they effectively reveal its grounds. These proofs are composed not of standard inferential steps, but of what we call grounding steps.

Whilst in contemporary logic proofs-why have received relatively little attention, proofs-that have been widely studied in the last century. Consider for example the natural deduction calculus introduced by Gentzen in 1935 which is composed by rules of the form:

$$
\frac{A_{1}, \ldots A_{n}}{B}
$$

Such a type of rule is standardly read in the following way: given that the premisses $A_{1}, \ldots A_{n}$ are true, then one can conclude that $B$ is true as well. Chained together, these rules constitute derivations. Thus derivations represent the logical counterpart of proofs-that. ${ }^{3}$

A central idea in our approach is to adapt Gentzen's model in order to formalize proofs-why. In particular we will introduce rules of the form

$$
\frac{C_{1}, \ldots C_{m}}{D}
$$

\footnotetext{
${ }^{2}$ Even if it is not spelled out in these terms, a similar idea can be found in [8].

${ }^{3}$ In a recent paper [15] Rumberg has put forward the view according to which normal derivations bear a strong resemblance to Bolzano's grounding-trees for conceptual truths. Since Bolzanian grounding trees correspond to proofs-why, the links between the present paper and Rumberg's work seem to be deep and interesting. However, since such a comparison would be quite long and beyond the scope of this work, we leave it for future research.
} 
These rules should be read in the following way: not only the truth of $D$ can be inferred from the the truth of the premisses $C_{1}, \ldots C_{m}$ but it is also grounded by them. In other terms, our rules are grounding rules in that they formalize grounding steps: they do not only establish the truth of their conclusion, more importantly, they also disclose its grounds. In the natural deduction calculus the symbol $\vdash$ is used to denote the passage from the premisses $A_{1}, \ldots A_{n}$ to $B$ and is standardly called derivability. Analogously we shall use the meta-linguistic symbol $\mid \sim$ to denote the grounding step from $C_{1}, \ldots C_{m}$ to $D$; it will be called formal explanation.

We shall thus use a meta-linguistic symbol to formalize proofs-why which in their turn track connection of formal grounding. However our analysis is not finished yet. Our logic for the notion of complete and immediate formal grounding will also contain the connective symbol $\triangleright$ which will be read in terms of because. ${ }^{4}$ There are at least three reasons for introducing such a connective. First of all, in this way it is possible to formulate introduction and elimination rules for the connective $\triangleright$ which are explicit, harmonious, symmetric and separated (see $[12,23])$. These properties are proof-theoretically significant (see [12, 23]), but so far mainly neglected by the existing literature on grounding. Secondly, the presence of the connective $\triangleright$ allows our logic of grounding to be better connected to other recent logics of grounding, many of which use a sentential operator (e.g. see $[5,9,18])$. Finally, thanks to the connective $\triangleright$, our logic of grounding retains an analogy with standard inferences. Indeed, in many logics derivability can be reflected in the language via the connective of material implication $\rightarrow{ }^{5}$ In the same way, in our logic of grounding the formal explanation relation $\mid \sim$ can be thought of as being reflected in the language via the sentential operator $\triangleright$.

Let us now move to analyse some features that our logic of grounding shares with prominent contemporary approaches $[4,5,9,18] .^{6}$

In Section 5 we establish a soundness and completeness result for the grounding part of our calculus. Note that such a result is not present in [18] nor in [9], while it is in $[4,5]$. We underline that, while in $[4,5]$ soundness and completeness are proved with respect to a semantics, in our case soundness and completeness are proved with respect to the definition of grounding given in [14]. In other

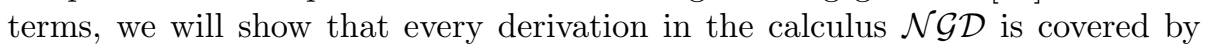
the definition of complete and immediate formal grounding given in [14], and that every case that is covered by the definition given in [14] is derivable in the

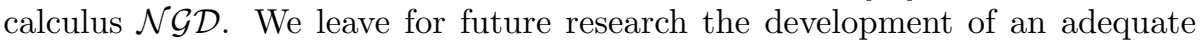
semantics for our logic.

There exists a deep link between the present work and the work of Correia [4]. Indeed one of the main characteristics of the logic that we propose is an account of ground-theoretic equivalence: we identify a subclass of logical equivalence that

\footnotetext{
${ }^{4}$ As we will explain in Section 4, after Definition 4.2, the connective $\triangleright$ does not entirely match the natural language term 'because'. Rather it is supposed to capture those uses of 'because' that correspond to the concept of grounding.

${ }^{5}$ This is formalized, for example in classical propositional logic, by the deduction theorem.

${ }^{6}$ We omit a comparison with the logic introduced in [10], since, contrary to ours, it is a purely structural logic for grounding.
} 
preserves the property of having the same grounds. While the logics introduced in $[5,9,18]$ are completely silent on this issue, a similar account is proposed and characterizes the logic illustrated in [4]. Thus this is a significant common point between the two approaches. There are also some important differences.

Firstly, while Correia conceptually defends the need of ground-theoretic equivalence, in the present paper this is a consequence of the chosen background framework (more precisely, it is the notion of $g$-complexity - see Definition 3.6 - that naturally leads to a notion of ground-theoretic equivalence). Secondly, while Correia introduces a symbol in his language to express factual equivalence, in our account the fact that a subset of formulas which are logically equivalent have the same grounds emerges within the grounding rules of the calculus, but never appears in the language. ${ }^{7}$ Finally, the principles that govern Correia's factual equivalence differ from those that can be found in the present account. For example, Correia's factual equivalence satisfies distribution $p \vee(q \wedge r) \leftrightarrow(p \vee q) \wedge(p \vee r),{ }^{8}$ while in our logic only associativity and commutativity of conjunction and disjunction are considered.

Let us end the section by mentioning a final point which concerns the structural features of grounding. The calculi proposed in $[4,9,18]$ contain rules, such as transitivity, irreflexivity and a-symmetry, which are supposed to describe the structural features of the grounding relation. In our approach, we will follow another strategy, which is the one mainly adopted in proof theory, e.g. see $[21,12]$ : our calculus will not contain any explicit structural rule; we will rather show that the relation of formal explanation enjoys certain structural properties, see Section 4, Proposition 4.8.

\section{The definition of complete and immediate for- mal grounding}

In this paper the main aim is to introduce a new propositional logic for the notion of complete and immediate formal grounding. As we will see, this logic will be based on a definition of the notion of complete and immediate formal grounding that we have introduced in [14]. We use this section to first briefly explain the intuitive idea behind this definition, and then to formally introduce it. Readers interested in a broad and deep philosophical explanation of the definition are referred to [14].

Roughly speaking, the definition of the notion of complete and immediate formal grounding given in [14] is based on two different ideas. The first consists in organizing all formulas of the language of grounding in a grounding hierarchy by means of the notion of $g$-complexity. Of particular interest are those formulas that belong to different levels of the hierarchy and are linked by the relation of being completely and immediately less g-complex.

\footnotetext{
${ }^{7}$ We believe this point to be directly linked with the previous one.

${ }^{8}$ The distribution principle has been questioned in the literature on grounding, e.g. see [11]. Note also that in a recent paper [6], Correia proposes a novel logic describing factual equivalence.
} 
Once all formulas are organized into the hierarchy, the task is to identify those formulas, enjoying the relation of being completely and immediately less g-complex, that stand in a grounding relation. This brings us to the second idea involved in the definition given in [14]. Consider three formulas $A, B$ and $C$ such that $A$ and $B$ are completely and immediately less g-complex than $C$. A grounding relation amongst $A, B$ and $C$ holds if, and only if, $A, B$ and $C$ satisfy two criteria that have been called positive and negative derivability. Positive derivability is simply standard derivability: in order to be in a grounding relation, $C$ must be derivable from $A$ and $B$. Negative derivability can be thought of as the complement of positive derivability: the negation of $C$ should also be derivable from the negation of $A$ and the negation of $B$. Positive and negative derivability, plus the relation of being completely and immediately less g-complex are thus the three key ingredients of the definition of immediate and complete formal grounding introduced in [14].

Note that, as underlined in the previous section, the account put forward in [14] involves a distinction between grounds and robust conditions. Robust conditions are strictly linked to negative derivability. Given three formulas $A, B$ and $C$ such that $A$ and $B$ are completely and immediately less g-complex than $C$, we will say that $A$ is a ground for $C$ under the robust condition $B$ if, and only if, $C$ is derivable from $A$, but also the negation of $C$ is derivable from the negation of $A$, and $B$.

Definition 3.1. The classical language $\mathcal{L}^{c}$ is composed of a denumerable stock of propositional atoms $(p, q, r, \ldots)$, the logical operators $\neg, \wedge$ and $\vee$, the parentheses (, ) and the square brackets [, ]. The connectives $\rightarrow$ and $\leftrightarrow$ are defined as usual; the symbol $\perp$ is defined as $A \wedge \neg A$. Propositional formulas are standardly constructed and their set is denoted by $\mathbb{P F}$.

Once the classical language $\mathcal{L}^{c}$, together with a set of propositional formulas $\mathbb{P F}$, is given, we can standardly define (e.g. by means of an Hilbert system, see $[22])$ the notion of classical derivability. From now on, we will write $M \vdash A$ to denote the fact that the formula $A$ is classically derivable from the multiset ${ }^{9}$ of formulas $M$.

Let us now move to the notion of g-complexity or, more precisely, the notion of being completely and immediately less g-complex. In order to define this notion, we first need to introduce other notions, namely that of converse of a formula and of subformula, and the relations of $a$-c equivalence and $\cong$. (The notion of converse of a formula and the relation $\cong$ will be directly used to define g-complexity; the notion of subformula, which is standard, serves to define the relation of $a$ - $c$ equivalence, which, in its turn, serves to define the relation $\cong$ ).

Definition 3.2. Let $D \in \mathbb{P F}$. The converse of $D$, written $D^{*}$, is defined in the following way

$$
D^{*}= \begin{cases}\neg^{n-1} E, & \text { if } D=\neg^{n} E \text { and } \mathrm{n} \text { is odd } \\ \neg^{n+1} E, & \text { if } D=\neg^{n} E \text { and } \mathrm{n} \text { is even }\end{cases}
$$

\footnotetext{
${ }^{9}$ We work with multisets of formulas rather than with sets of formulas because we need to take into account the number of occurrences of each formula of M.
} 
where the principal connective of $E$ is not a negation, $n \geqslant 0$ and 0 is taken to be an even number.

Let us provide some examples that help to clarify Definition 3.2. If $D=$ $\neg \neg \neg \neg p$, then its converse, $D^{*}$, is $\neg \neg \neg \neg \neg p$. If $D=\neg(A \wedge B)$, then its converse, $D^{*}$, is $(A \wedge B)$; finally, if $D=(A \vee B)$, then its converse, $D^{*}$, is $\neg(A \vee B)$. From now on we will use capital letters to refer to objects of $\mathbb{P F}$ and their converse.

Definition 3.3. Given $A$ and $B \in \mathbb{P F}$, we say that $A$ is a subformula of $B$ if, and only if:

$$
\begin{aligned}
& A=B, \\
& \text { or } B=\neg C \text { and } A \text { is a subformula of } C, \\
& \text { or } B=C \circ D^{10} \text { and } A \text { is a subformula of } C \text { or } A \text { is a subformula of } D
\end{aligned}
$$

By exploiting the standard notion of subformula, we introduce and define the relation $a$-c equiv. Intuitively, the relation $a-c$ equiv can be explained as follows; given $A$ and $B \in \mathbb{P F}$, we say that $A$ is $a$-c equiv to $B$ when $A$ is equivalent to $B$ by means of several applications of associativity and commutativity of conjunction and disjunction.

Definition 3.4. Given $A$ and $B \in \mathbb{P F}, A$ is a-c equiv to $B$ if, and only if:

$A=B$,

or $E \circ F$ is a subformula of $A$ and the formula obtained by substituting $F \circ E$ for $E \circ F$ in $A$ is a-c equiv to $B$,

or $(E \circ F) \circ G$ is a subformula of $A$ and the formula obtained by substituting $E \circ(F \circ G)$ for $(E \circ F) \circ G$ in $A$, is a-c equiv to $B$.

Let us provide some exemples of formulas $A$ and $B$ such that $A$ is $a$ - $c$ equiv to $B . A \wedge(B \wedge C)$ is $a-c$ equiv to $C \wedge(A \wedge B) . \neg((E \vee F) \wedge(G \wedge(H \vee D)))$ is $a-c$ equiv to $\neg((F \vee E) \wedge(G \wedge(D \vee H)))$, but also to $\neg((G \wedge(D \vee H)) \wedge(F \vee E))$. $A \wedge((B \vee C) \vee(D \vee E))$ is a-c equiv to $A \wedge((D \vee B) \vee(E \vee C))$.

Definition 3.5. Given $A, B \in \mathbb{P F}, A \cong B$ if, and only if:

$A$ is a-c equiv to $B$ or $A$ is a-c equiv to $B^{*}$

As extensively discussed in [14], two formulas $A$ and $B$ stand in the relation denoted by $\cong$ when they are about, or pertain to, or concern the same issue. The relation $\cong$ is thus analogous (though not equivalent) to the notion of factual equivalence discussed in [5].

Definition 3.6. Given a multiset of formulas $M$ and a formula $C \in \mathbb{P F}$, we say that $M$ is completely and immediately less $g$-complex than $C$, if, and only if:

${ }^{10}$ The symbol $\circ \in\{\wedge, \vee\}$. 
- $C \cong \neg \neg B$ and $M=\{B\}$ or $M=\left\{B^{*}\right\}$, or

- $C \cong(B \circ D)$ and, $M=\{B, D\}$ or $M=\left\{B^{*}, D\right\}$ or $M=\left\{B, D^{*}\right\}$ or $M$ $=\left\{B^{*}, D^{*}\right\}$.

Let us provide some examples that help to clarify this notion. The multisets $\{(p \wedge q) \wedge r\},\{\neg((q \wedge p) \wedge r)\},\{(r \wedge p) \wedge q\},\{\neg(q \wedge r) \wedge p\}$ are all completely and immediately less g-complex than $\neg \neg(r \wedge(q \wedge p))$. The multisets $\{(p \vee q), r\}$, $\{(q \vee p), r\},\{\neg(q \vee p), r\},\{\neg(p \vee q), \neg r\}$ are all completely and immediately less g-complex than $\neg((p \vee q) \wedge r)$.

Definition 3.7. For any consistent multiset of formulas $C \cup M$ such that $C$ and $M \in \mathbb{P F}$, we say that, under the robust condition $C$ (that may be empty), $M$ completely and immediately formally grounds $A$, in symbols $[C] M \mid \sim \mathrm{A}$, if and only if:

- $M \vdash A$

- $C, \neg(M) \vdash \neg A$

- $C \cup M$ is completely and immediately less g-complex than $A$ in the sense of Definition 3.6.

where $\neg(M):=\{\neg B \mid B \in M\}$.

Under the robust condition $C$, the multiset $M$ completely and immediately formally grounds $A$ if, and only if, (i) $A$ is derivable from $M$ - positive derivability; (ii) $\neg A$ is derivable from $\neg(M)$ plus $C$ - negative derivability; (iii) $C \cup M$ is completely and immediately less g-complex than $A$.

In the following section we will introduce a logic for the notion of complete and immediate formal grounding, which has the form of the calculus of natural

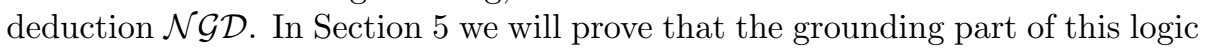
is sound and complete with respect to the definition just given.

\section{The calculus NGD}

Definition 4.1. The language $\mathcal{L}^{g}$ extends the classical language $\mathcal{L}^{c}$ (see Definition 3.1) by means of the completely and immediately because connective $\triangleright$. As before, $M, N, \ldots$ denote multisets of formulas; the notation $\bigwedge(\neg M)$ stands for the conjunction of the negation of each element of $M$.

Definition 4.2. Let $\mathbb{B}$ be the set of completely and immediately because formulas defined in the following way:

$$
\begin{aligned}
\mathbb{B}:= & \{A \triangleright B \mid A, B \in \mathbb{P} \mathbb{F}\} \cup\{A, C \triangleright B \mid A, B, C \in \mathbb{P F}\} \cup \\
& \{[C] A \triangleright B \mid A, B, C \in \mathbb{P F}\}
\end{aligned}
$$


Figure 1: Rules of the $\mathcal{N D}$ part

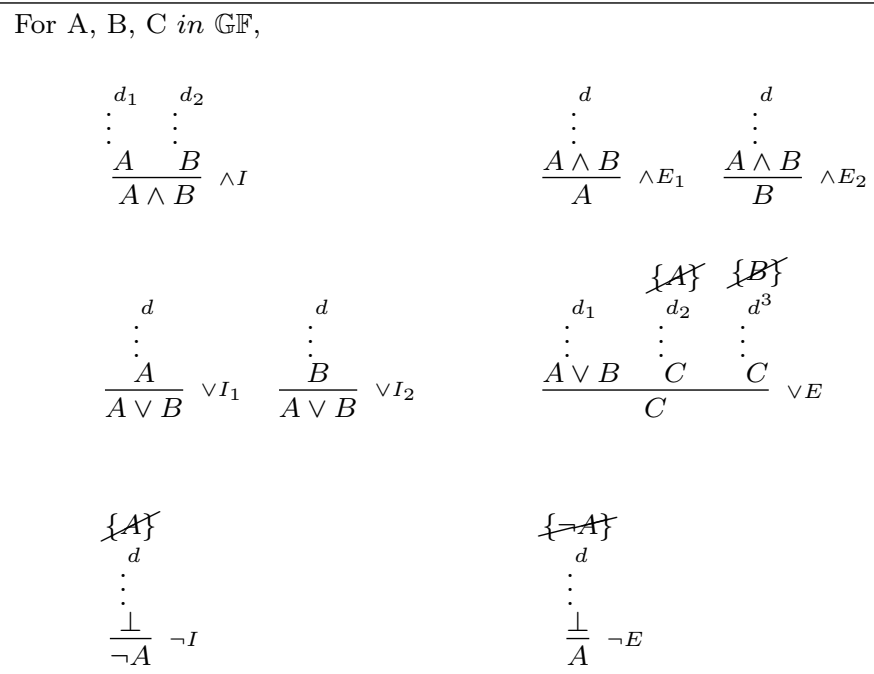

First of all, let us note that the connective $\triangleright$ together with the set $\mathbb{B}$ is specifically defined for propositional logic. Thus, if one aims at using $\triangleright$ and $\mathbb{B}$ for treating quantifiers, their definition needs to be extended. Secondly, the connective $\triangleright$ is not supposed to match the natural-language use of the term because; it is rather supposed to capture those uses of 'because' that correspond to the concept of grounding. Thirdly, while in the natural language use of the term 'because' the explanans come after the 'because' and the explanandum before, in the connective $\triangleright$ these positions are inverted. Fourthly, because of the points just raised, the connective $\triangleright$ is quite different from the 'becauseconnectives' used in other logics of grounding, see in particular [18].

We will denote with $\mathbb{G F}$ the closure of $\mathbb{P F} \cup \mathbb{B}$ under the propositional connectives of $\mathcal{L}^{g}$.

Based on the language $\mathcal{L}^{g}$, we will construct the calculus $\mathcal{N G D}$ which can be thought of as divided in three parts:

(i) the first part, the $\mathcal{N D}$ part, which is the basis of the calculus, is just the classical natural deduction calculus. It is composed of the standard introduction and elimination rules which characterize the classical relation of derivability, denoted by $\vdash$, that we have already introduced in the previous section.

(ii) the second part of the calculus, the $\mathcal{N G}$ part, is where the grounding concept is analyzed and studied; grounding rules define the relation of complete and immediate formal explanation, denoted by $\mid \sim{ }^{11}$

${ }^{11}$ Note that we use the same symbol for the relation of complete and immediate formal 
(iii) the third part of the calculus, the $\mathcal{N}$ part, is where the connective $\triangleright$ is proof-theoretically defined by means of one introduction rule and three elimination rules. The introduction rule naturally provides a link between parts (i) and (ii) of the calculus. Thanks to the $\mathcal{N}$ part of the calculus, the relation $\mid \bumpeq$, that holds for the whole logic $\mathcal{N G D}$ and which combines the classical notion of derivability with the notion of complete and immediate formal explanation, can finally be defined.

For the sake of clarity, we will introduce each part separately. Let us start by (i). The $\mathcal{N D}$ part of the calculus is composed by the rules of Figure 1, which are the rules of the classical natural deduction calculus. As is standard, in these rules the curly brackets stand for the discharge of several formulas at the same time; in the rule $\neg I$ the $A$ might be empty. The set of derivations $\mathbb{C D}$ that can be constructed by means of the rules of $\mathcal{N D}$ is the standard one, see [22]. We write $M \vdash_{\mathcal{N D}} A$ to denote the fact that there exists a natural deduction derivation from the multiset $M$ to $A$ in $\mathcal{N D}$. We assume each of the rules of $\mathcal{N D}$ to be applicable not only on formulas standardly written, but also on formulas written in square brackets. At this level of the calculus, the difference is a mere question of notation; nevertheless, it shall prove useful in the proof of Lemma 4.5 .

In order to introduce part (ii) of the calculus, we need some preliminary notions and definitions.

Definition 4.3. Given $A \in \mathbb{P F}$, let $\mathbb{A} \mathbb{C}(A)$ be the set of all elements $B$ such that $A$ is a-c equiv to $B$ (Definition 3.4). From now on we will use $\mathbf{A}, \mathbf{B}, \mathbf{C}$, ... to denote generic elements belonging to the set $\mathbb{A} \mathbb{C}(A), \mathbb{A} \mathbb{C}(B), \mathbb{A} \mathbb{C}(C), \ldots$ respectively.

Definition 4.4. Let $\mathbf{A}$ be a generic element belonging to the set $\mathbb{A} \mathbb{C}(A)$. Then $\mathbf{A}^{*}$ denotes generic elements of $\mathbb{A} \mathbb{C}\left(A^{*}\right)$.

Let us illustrate our notation with some examples. Consider an object $A$ of the form $\neg((p \wedge q) \vee r)$, then

A denotes any of the following objects: $\neg((p \wedge q) \vee r), \neg((q \wedge p) \vee r), \neg(r \vee(p \wedge q))$, $\neg(r \vee(q \wedge p))$

A* denotes any of the following objects: $(p \wedge q) \vee r,(q \wedge p) \vee r, r \vee(p \wedge q)$, $r \vee(q \wedge p)$

From now on, capital letters of bold character will be used to refer to both $\mathbf{A}, \mathbf{B}, \mathbf{C}, \ldots$ but also $\mathbf{A}^{*}, \mathbf{B}^{*}, \mathbf{C}^{*}, \ldots \mathbf{M}, \mathbf{N}, \ldots$ will be multisets of capital letters

explanation and the relation of complete and immediate formal grounding, introduced in Definition 3.7. Though this might at first appear confusing, both notions are syntactic and will be proved to be equivalent, so we prefer to leave the notation as it is. This is analogous to what happens in Hilbert systems and Gentzen systems: they share the same symbol for derivability. 
Figure 2: Bottom Rules
$\frac{\mathbf{A} \quad \neg A}{\perp} \perp F$
$\frac{\mathbf{A}^{*} \quad A}{\perp} \perp^{*}$
$\frac{[\mathbf{A}] \quad\left[A^{*}\right]}{\perp} \perp[]$

of bold character. Analogously to what we have seen in the previous section, [D] will indicate the robust condition D. Capital letters in bold character play a crucial role in the formulation of the rules of the $\mathcal{N G}$ part of the calculus.

Lemma 4.5. Each of the rules $\perp F, \perp^{*}$ and $\perp^{[]}$(see Figure 2) is derivable in the $\mathcal{N D}$ part of the calculus.

Proof. We start analyzing the rule $\perp F$. Suppose that in $\perp F \mathbf{A}$ denotes an object $A^{\prime} \in \mathbb{A} \mathbb{C}(A)$. From the way the set $\mathbb{A} \mathbb{C}(A)$ has been defined, we know that there exists a derivation $d$ from $A^{\prime}$ to $A$; we apply to such a derivation $d$ the rule $\wedge I$ with $\neg A$ as other premise and we obtain the desired result.

For the sake of clarity we give a concrete example of what we have just said. Assume that $A=(r \wedge q) \wedge p$, then we show that the following instance of the rule $\perp F$

$$
\frac{(p \wedge q) \wedge r \quad \neg((r \wedge q) \wedge p)}{\perp}
$$

is derivable in the $\mathcal{N D}$ part of our calculus. We have:

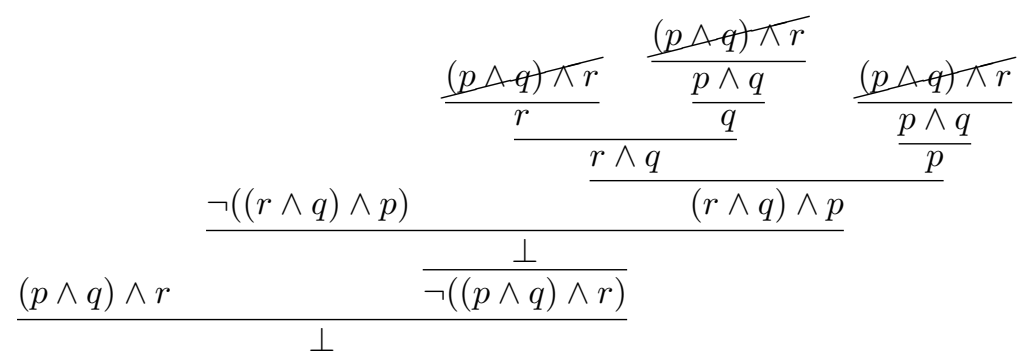

As for the derivability of the rules $\perp^{*}$ and $\perp^{[]}$, the procedure is analogous. In case of the rule $\perp^{[}$, the only difference involves the fact that formulas should be framed in square brackets (as already emphasized, this is just a notational difference). For the sake of clarity we give a concrete example of the derivability of $\perp^{[]}$. Assume that $[A]=[(p \vee q) \vee r]$ and $\left[A^{\prime}\right]=[(r \vee q) \vee p]$; then $\left[\left(A^{\prime}\right)^{*}\right]=$ $[\neg((r \vee q) \vee p)]$. We show that the following instance of the rule $\perp^{[]}$

$$
\frac{[(p \vee q) \vee r] \quad[\neg((r \vee q) \vee p)]}{\perp}
$$

is derivable in the $\mathcal{N D}$ part of our calculus. We have (for the sake of space we write $X$ instead of $[\neg((r \vee q) \vee p)])$ : 
Figure 3: Rules of the $\mathcal{N G}$ part

For A, B, C in $\mathbf{P F}^{+}$

$\mathbf{A}, \mathbf{B}$

$\widetilde{\mathrm{A} \wedge \mathrm{B}}$
A, B
$\left[\mathrm{B}^{*}\right] \mathbf{A}$
$\left[\mathbf{A}^{*}\right] \mathbf{B}$
$\widehat{\mathbf{A} \vee \mathbf{B}}$
$\widetilde{\mathbf{A} \vee \mathbf{B}}$
$\widehat{A \vee B}$

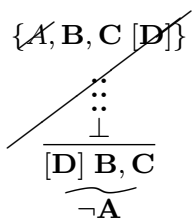

All rules carry the proviso that their premisses are consistent.

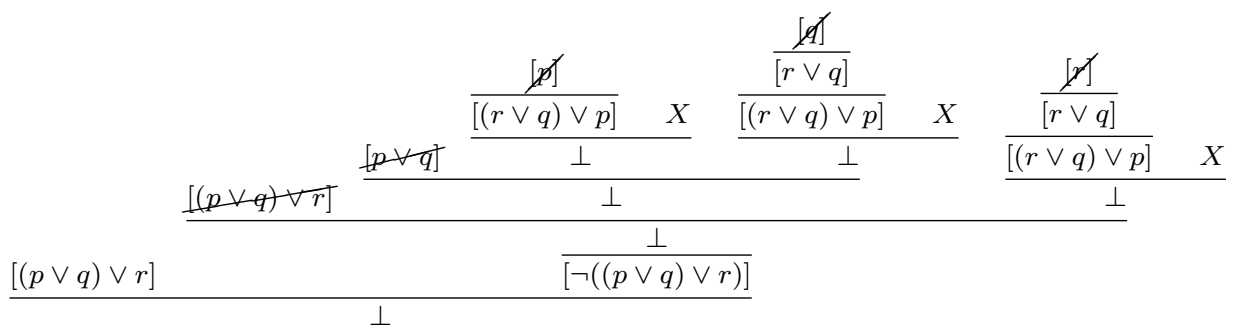

The rules of Figure 2 play a crucial role in the following definition.

Definition 4.6. $\{A, \mathbf{B}, \mathbf{C}[\mathbf{D}]\}$, where $\mathbf{D}$ might be empty and $\mathbf{B}$ and $\mathbf{C}$ might be reduced to a single element, is said to be completely and immediately contradictory if, and only if, there exists a derivation $d$ from $\{A, \mathbf{B}, \mathbf{C}[\mathbf{D}]\}$ to $\perp$ that has one of the following forms:

$$
\begin{array}{cl}
\mathrm{d}:= & \perp F \\
\mathrm{~d}:= & \stackrel{\perp}{*}^{*} E^{\perp^{*}} \\
& \wedge E_{i} \\
& \perp^{*} \\
\neg & I \\
\mathrm{~d}:=\perp^{[]} / \perp^{*}
\end{array}
$$

where the last item carries the following proviso: if the last rule is $\perp^{*}$, then the formula introduced by $\neg I$ is the conjunct which has not been eliminated by the rule $\wedge E_{i}$ above. If the last rule is $\perp^{[]}$, then the object in square brackets 
introduced by $\neg I$ is the converse of the conjunct which has not been eliminated by the rule $\wedge E_{i}$ above. The notation:

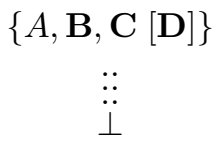

serves to indicate that $\{A, \mathbf{B}, \mathbf{C}[\mathbf{D}]\}$ is completely and immediately contradictory.

We now have all elements to introduce part (ii) of the calculus, namely the $\mathcal{N G}$ part. The $\mathcal{N G}$ part is composed of the rules of Figure 3. The rule describing the complete and immediate formal explanans of a conjunction is called $\wedge G$; the three rules providing the complete and immediate formal explanans of a disjunction are called $\vee G_{1}, \vee G_{2}, \vee G_{3}$, respectively; finally, the rule concerning negation is called $\neg G$. Note that in the rule $\neg G, \mathbf{B}, \mathbf{C}$ and $\mathbf{D}$ all occur twice. We assume that both the occurrences of each of them denote the same element of $\mathbb{A} \mathbb{C}(B), \mathbb{A} \mathbb{C}(C)$ and $\mathbb{A} \mathbb{C}(D)$, respectively. As for $\neg \mathbf{A}$, this can denote any object $A^{\prime}$ belonging to $\mathbb{A} \mathbb{C}(\neg A)$.

Definition 4.7. The set of complete and immediate formal explanations of the calculus $\mathcal{N G D}$ is the smallest set $\mathbb{F} \mathbb{E}$ of derivations using only rules of Figure 3. We will write $[\mathbf{C}] \mathbf{M} \mid \sim \mathbf{A}$ for: under the robust condition $\mathbf{C}$ there exists a complete and immediate formal explanation of $\mathbf{A}$ from $\mathbf{M}$. We will also use the notation

\section{$[\mathbf{C}] \mathbf{M}$ \\ ? \\ A}

to indicate that $\mathbf{A}$ is linked to $[\mathbf{C}] \mathbf{M}$ by means of one of the rules of Figure 3.

Let us provide some examples of complete and immediate formal explanations. Here is a formal explanation using the rule $\wedge G$ :

$$
\begin{aligned}
& p \wedge(q \wedge r), \quad s \vee t \\
& (t \vee s) \wedge(r \wedge(p \wedge q))
\end{aligned}
$$

The following two are formal explanations using the rules $\vee G_{2} / \vee G_{3}$.

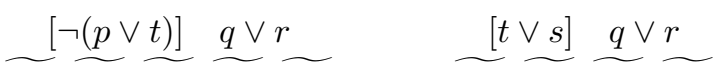

$$
\begin{aligned}
& (p \vee q) \vee(r \vee t) \quad \neg(t \vee s) \vee(r \vee q)
\end{aligned}
$$

We now introduce two formal explanations that involve the rule $\neg G$ : 

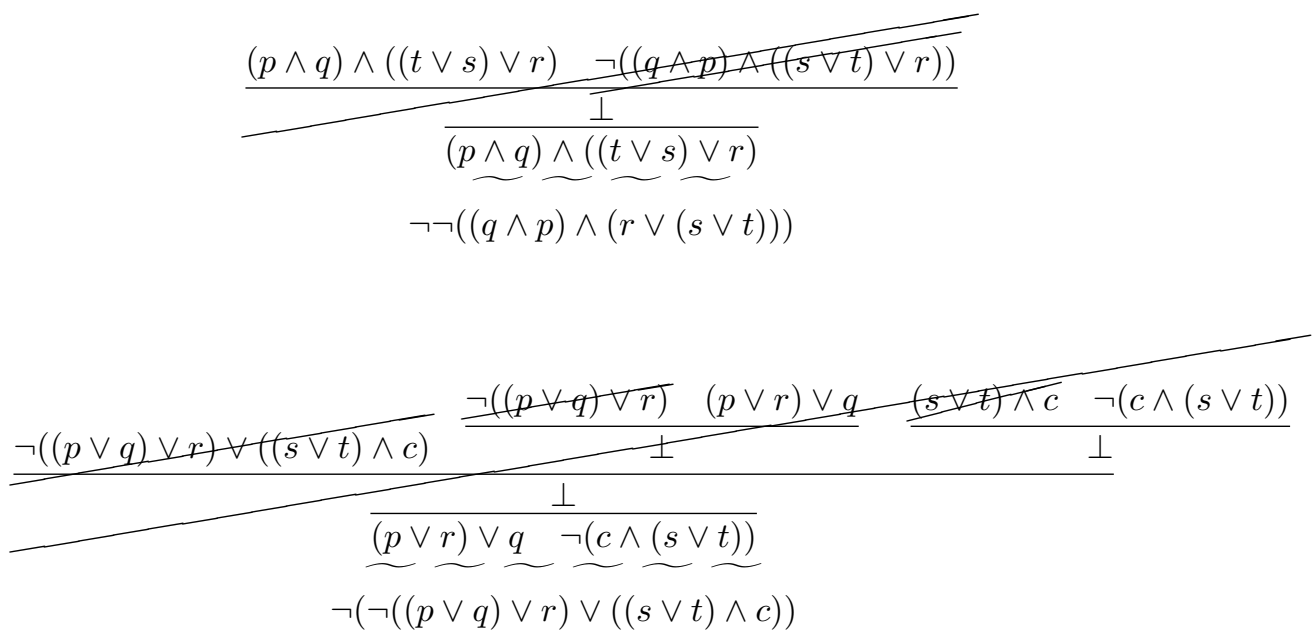

Since in this paper we are only interested in a logic for the notion of complete and immediate ground, our grounding rules cannot form grounding chains.

Let us emphasize some important structural properties of the relation of complete and immediate formal explanation.

Proposition 4.8. The relation $\mid \sim$ is not reflexive, nor monotonic, nor transitive, nor symmetric.

Proof. The fact that the relation $\mid \sim$ is not reflexive and not symmetric are evident. The relation $\mid \sim$ is non-monotonic and not transitive because both monotonicity and transitivity would break the g-complexity constraint between premisses and conclusion.

We thus have introduced the first two parts of our calculus: the $\mathcal{N D}$ part with the related notion of classical derivability and the $\mathcal{N G}$ part with the related crucial notion of formal explanation. We now introduce the third and last part

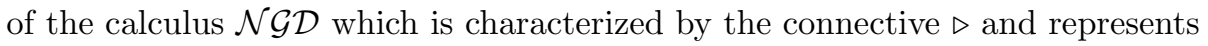
the bridge between the first two parts.

The $\mathcal{N}$ part of our calculus is composed by the rules of Figure 4 . These rules introduce and eliminate the connective $\triangleright$ which is supposed to be the linguistic counterpart of the relation of complete and immediate formal explanation and which should be read as completely and immediately because. Let us consider in detail the rules $\triangleright I, \triangleright E_{1}, \triangleright E_{2}, \triangleright E_{3}$ that compose the third part of our calculus. As concerns the rule $\triangleright I$, let us note the similarity between this rule and the rule that standardly introduces the implication connective, namely

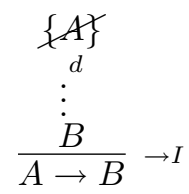


Figure 4: Rules of the $\mathbf{N}$ part

For A, C, M in $\mathbf{P F}^{+}$,

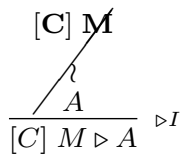

$$
\begin{aligned}
& \frac{[C] M \triangleright A}{\wedge M \rightarrow A} \triangleright E_{1} \\
& \frac{[C] M \triangleright A}{C \wedge \wedge(\neg M) \rightarrow \neg A} \triangleright E_{2} \\
& \frac{[C] M \triangleright A}{\perp} \triangleright E_{3}
\end{aligned}
$$

The rule $\triangleright E_{3}$ can be applied only in case the multiset $C \cup M$ is inconsistent or is not completely and immediately less g-complex than $A$, according to

Definition 3.6.

As the rule $\rightarrow I$ states that, if there exists a derivation from $A$ to $B$, then $A \rightarrow B$ can be inferred, in the same way, the rule $\triangleright I$ states that, if there exists a complete and immediate formal explanation from $M$ to $A$, then $M \triangleright A$ can be inferred. In other terms, this similarity reflects the analogy between the relation between derivability and material implication on the one hand, and the relation between formal explanation and 'because' on the other hand.

Let us now turn to the three elimination rules that concern the connective $\triangleright$. Taken together, these rules give a precise interpretation of the connective $\triangleright$. They say that if, under the robust condition $C, A$ completely and immediately because of $M$, then: (i) the conjunction of each element of $M$ implies $A$, (ii) the negation of each element of $M$ together with $C$ implies the negation of $A$; (iii) $C \cup M$ is a consistent multiset and is completely and immediately less g-complex than $A$. Conditions (i)-(iii) reflect at the linguistic level the three conditions that characterize the notion of formal explanation introduced in Definition 3.7. In particular, (i) corresponds to positive derivability, (ii) to negative derivability, and (iii) is basically the same condition as complexity (and consistency).

The calculus $\mathcal{N G D}$ is thus composed of the rules of Figure 1, the rules of Figure 3 and the rules of Figure 4 . The notion of derivability that holds for the

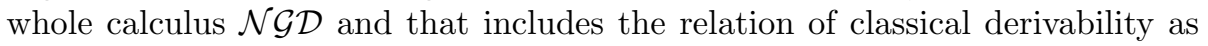
well as that of formal explanation can be defined as follows.

Definition 4.9. The set of $\mathcal{N G D}$ derivations is the smallest set $\mathbb{G D}$ such that:

(1) For all $A \in \mathbb{G} \mathbb{F}$ (see Definition 4.2), $A$ belongs to $\mathbb{G} \mathbb{D}$.

(2) Each derivation $d \in \mathbb{C D}$ and each formal explanation $f \in \mathbb{F} \mathbb{E}$ belongs to $\mathbb{G D}$. 
(3) Each application of the rule $\triangleright I$ to a formal explanation in $\mathbb{F} \mathbb{E}$ belongs to $\mathbb{G D}$.

$(4 \wedge)$ If ${ }_{A}^{d_{1}}$ and ${ }_{B}^{d_{2}} \in \mathbb{G D}$ and, in case $d_{1}$ and $d_{2}$ contain complete and immediate formal explanations discharged by the rule $\triangleright I$, and the premisses of these formal explanations form a consistent multiset, then ${ }_{A}^{d_{1}}$ and ${ }_{B}^{d_{2}}$ followed (and united) by one application of the rule $\wedge I$ belong to $\mathbb{G D}$.

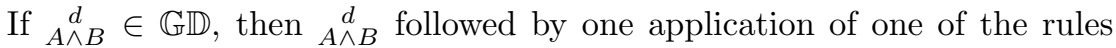
$\wedge E_{i}, i \in\{1,2\}$, belongs to $\mathbb{G D}$.

$(5 \vee)$ If ${ }_{A}^{d} \in \mathbb{G D}$, then ${ }_{A}^{d}$ followed by one application of the rule $\vee I$ belongs to $\mathbb{G D}$. The same holds for $\underset{B}{d}$.

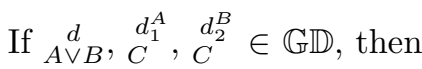

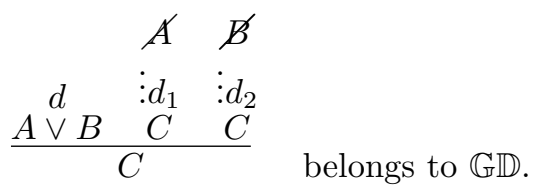

$(6 \neg)$ if $\underset{\perp}{d^{A}} \in \mathbb{G D}$, then

$A$
$\vdots$
$\frac{\perp}{\neg A}$ belongs to $\mathbb{G D}$.

If $\underset{\perp}{d_{\perp}^{A}} \in \mathbb{G D}$, then

$\neg A$

$\vdots_{d}$

$\stackrel{\perp}{A}$

belongs to $\mathbb{G D}$.

From now on we will write $M \mid \backsim A$ for: there exists a derivation belonging to the set $\mathbb{G D}$ from the multiset of formulas $M$ to the formula $A$.

Let us first emphasize two significant points of the definition just given and

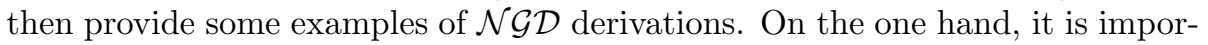
tant to note that, according to Definition 4.9, a formal explanation is linked to a derivation only by means of the rule $\triangleright I$; in other words, none of the classical rules of introduction and elimination (see Figure 1) can be directly applied to a formal explanation. On the other hand, it is crucial to stress the importance of the condition of consistency of the premisses for the application of the rule $\wedge I$ (see $(4 \wedge))$ to derivations containing formal explanations. This condition prevents cases such as the following one: 


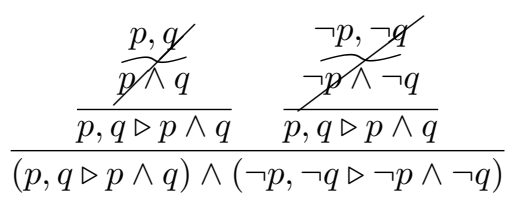

This derivation proves the theorem $(p, q \triangleright p \wedge q) \wedge(\neg p, \neg q \triangleright \neg p \wedge \neg q)$ that cannot be accepted in a reasonable logic of grounding, since grounding is a relation (or a sentential operator) amongst truths and $p, q, \neg p, \neg q$ cannot all be true at the same time. The consistency clause prevents this type of derivation.

We now give two paradigmatic examples of $\mathcal{N G D}$ derivations. First of all, it can be easily proved that it is not the case that $A$ completely and immediately because of $\neg A$.

$$
\frac{\neg A \triangleright A}{\neg(\neg A \triangleright A)}
$$

Then it can be proved that $\neg(\neg p \vee \neg q)$ completely and immediately because of $p$ and $q$.

$$
\frac{\neg(\neg p \vee \neg q)}{p, q \triangleright \neg(\neg p \vee \neg q)}
$$

\section{Soundness and Completeness}

We start this section by briefly summarizing the developments up to this point. In the last two sections we have introduced the calculus $\mathcal{N G D}$ together with its derivability relation $\mid \simeq$ and its set of derivations $\mathbb{G D}$. The calculus $\mathcal{N G D}$ is composed of three parts: first we have the $\mathcal{N D}$ part of the calculus together with the classical derivability relation $\vdash$ and the set of classical derivations $\mathbb{C D}$; secondly, we have the $\mathcal{N G}$ part of the calculus together with the formal explanation relation $\mid \sim$ and the set of formal explanations $\mathbb{F E}$; finally, we have the $\mathcal{N}$ part of the calculus that serves to define the operator $\triangleright$ and connects the first two parts $\mathcal{N D}$ and $\mathcal{N G}$. Note that, just as the calculus $\mathcal{N G D}$ can be thought of as being composed of three parts, analogously its set of derivations $\mathbb{G D}$ contains the set of derivations $\mathbb{C D}$ and $\mathbb{F E}$, and its derivability relation $\models \simeq$ results from the combination of the classical derivability relation $\vdash$ and the formal explanation relation $\mid \sim$ (see Figure 5).

In this section we will focus on the $\mathcal{N G}$ part of the calculus and we will prove that this part is sound and complete with respect to the notion of complete and immediate formal grounding that has been defended in [14] and recalled in Definition 3.7. In other words, in this section we will show that the grounding part of our logic $\mathcal{N G D}$ is the adequate formal counterpart of the conception 
Figure 5: The calculus $\mathcal{N G D}$

$$
\mathcal{N G D}, \mid \simeq, \mathbb{G D}=\left\{\begin{array}{l}
\mathcal{N D}, \vdash, \mathbb{C D} \\
\mathcal{N G}, \mid \sim, \mathbb{F E} \\
\mathcal{N}
\end{array}\right.
$$

of grounding put forward in [14]. Note that soundness and completeness do not need to be shown for the $\mathcal{N D}$ part of the calculus since this is a standard result, e.g. see [22]. The only part that would also call for a soundness and completeness result is the $\mathcal{N}$ part. Following the strategy of other logicians of grounding (see $[4,18]$ ), we will leave this task for further research.

Lemma 5.1. For any completely and immediately contradictory multiset $\{A, \mathbf{B}, \mathbf{C}[\mathbf{D}]\}$, it holds that, not only $A, \mathbf{B}, \mathbf{C} \vdash \perp$, but also $\neg A, \neg \mathbf{B}, \neg \mathbf{C}, \mathbf{D} \vdash \perp$. Moreover $\{\mathbf{B}, \mathbf{C}, \mathbf{D}\}$ is completely and immediately less $g$-complex than $\neg A$ according to the notion of g-complexity introduced in Definition 3.6.

Proof. We prove this lemma by distinguishing the several forms that the derivation from $\{A, \mathbf{B}, \mathbf{C}[\mathbf{D}]\}$ to $\perp$ might have (see Definition 4.6) and thus the several types the multiset $\{A, \mathbf{B}, \mathbf{C}[\mathbf{D}]\}$ might be.

(1) $\mathrm{d}:=\perp F$ and thus it has the form,

$$
\frac{\mathbf{A}, \neg A}{\perp}
$$

Hence our multiset is $\{\neg A, \mathbf{A}\}$. Suppose $\mathbf{A}$ denotes an object $A^{\prime} \in \mathbb{A} \mathbb{C}(A)$ (the case where $\mathbf{A}$ denotes $A$ is trivial). By the way we have defined the set $\mathbb{A} \mathbb{C}(A)$, we have that $A^{\prime} \vdash A$; since, by logic, it holds that $A, \neg A \vdash \perp$, we have $A^{\prime}, \neg A \vdash \perp$. Moreover, from the fact that $A^{\prime}, \neg A \vdash \perp$ holds, it follows that $\neg A^{\prime}, \neg \neg A \vdash \perp$ holds too.

Finally it is easy to see that $\neg \neg A \cong \neg \neg A^{\prime}$ and thus $\left\{A^{\prime}\right\}$ is completely and immediately less complex than $\neg \neg A$.

(2) $\mathrm{d}:={ }^{\perp^{*}} \vee E^{\perp^{*}}$ and so we have,

$$
\frac{A \vee B \frac{A, \mathbf{A}^{*}}{\perp} \frac{\not B, \mathbf{B}^{*}}{\perp}}{\perp}
$$

Hence our multiset is $\left\{A \vee B, \mathbf{A}^{*}, \mathbf{B}^{*}\right\}$. Suppose $\mathbf{A}^{*}$ denotes an object $\left(A^{\prime}\right)^{*}$ such that $A^{\prime} \in \mathbb{A} \mathbb{C}(A)$ and $\mathbf{B}^{*}$ denotes an object $\left(B^{\prime}\right)^{*}$ such that $B^{\prime} \in \mathbb{A} \mathbb{C}(B)$. By the 
way we have defined the sets $\mathbb{A} \mathbb{C}(A)$ and $\mathbb{A C}(B)$ and the notion of converse of an object, we have that: (i) $\left(A^{\prime}\right)^{*} \vdash \neg A$ and $\left(B^{\prime}\right)^{*} \vdash \neg B$; and (ii) $\neg\left(\left(A^{\prime}\right)^{*}\right) \vdash A$ and $\neg\left(\left(B^{\prime}\right)^{*}\right) \vdash B$. From (i) and $A \vee B, \neg A, \neg B \vdash \perp$, which holds by logic, we get $A \vee B,\left(A^{\prime}\right)^{*},\left(B^{\prime}\right)^{*} \vdash \perp$. From (ii) and $\neg(A \vee B), A, B \vdash \perp$, which holds by logic, we get $\neg(A \vee B), \neg\left(\left(A^{\prime}\right)^{*}\right), \neg\left(\left(B^{\prime}\right)^{*}\right) \vdash \perp$. Finally, it is easy to see that $\neg(A \vee B) \cong \neg\left(A^{\prime} \vee B^{\prime}\right)$; hence $\left\{\left(A^{\prime}\right)^{*},\left(B^{\prime}\right)^{*}\right\}$ is completely and immediately less g-complex than $\neg(A \vee B)$.

$$
\begin{gathered}
\wedge E_{i} \\
\perp^{*} \\
\neg I
\end{gathered}
$$

(3) $\mathrm{d}:=\perp^{[]} / \perp^{*}$ and so we have the following four possibilities,
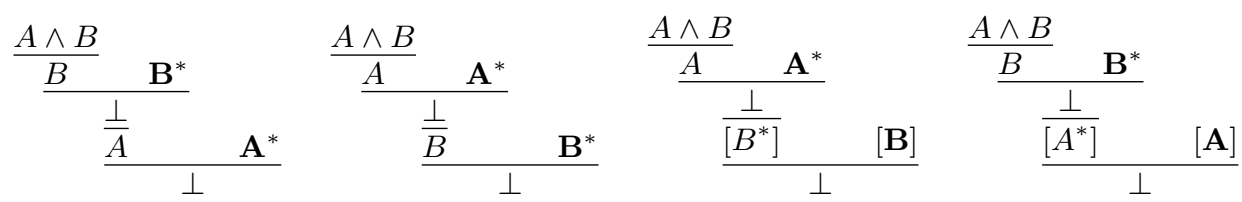

Hence there are the following three multisets: $\left\{A \wedge B, \mathbf{A}^{*}, \mathbf{B}^{*}\right\},\left\{A \wedge B, \mathbf{A}^{*}[\mathbf{B}]\right\}$, $\left\{A \wedge B, \mathbf{B}^{*}[\mathbf{A}]\right\}$. For the analysis of these three multisets, we suppose that $\mathbf{A}^{*}$ denotes the object $\left(A^{\prime}\right)^{*}$ such that $A^{\prime} \in \mathbb{A} \mathbb{C}(A)$, and $\mathbf{B}^{*}$ denotes the object $\left(B^{\prime}\right)^{*}$ such that $B^{\prime} \in \mathbb{A} \mathbb{C}(B)$.

Let us first analyse the multiset $\left\{A \wedge B, \mathbf{A}^{*}, \mathbf{B}^{*}\right\}$. By the way we have defined the sets $\mathbb{A C}(A)$ and $\mathbb{A C}(B)$ and the notion of converse of an object, we have that: (i) $\left(A^{\prime}\right)^{*} \vdash \neg A$ and $\left(B^{\prime}\right)^{*} \vdash \neg B$; and (ii) $\neg\left(\left(A^{\prime}\right)^{*}\right) \vdash A$ and $\neg\left(\left(B^{\prime}\right)^{*}\right) \vdash B$. From (i) and $A \wedge B, \neg A, \neg B \vdash \perp$, which holds by logic, we get $A \wedge B,\left(A^{\prime}\right)^{*},\left(B^{\prime}\right)^{*} \vdash \perp$. From (ii) and $\neg(A \wedge B), A, B \vdash \perp$, which holds by logic, we get $\neg(A \wedge B), \neg\left(\left(A^{\prime}\right)^{*}\right), \neg\left(\left(B^{\prime}\right)^{*}\right) \vdash \perp$. Finally, it is easy to see that $\neg(A \wedge B) \cong \neg\left(A^{\prime} \wedge B^{\prime}\right)$; hence $\left\{\left(A^{\prime}\right)^{*},\left(B^{\prime}\right)^{*}\right\}$ is completely and immediately less g-complex than $\neg(A \wedge B)$.

Let us move to the analysis of the second multiset $\left\{A \wedge B, \mathbf{A}^{*}[\mathbf{B}]\right\}$. From $A \wedge B, \neg A \vdash \perp$, which holds by logic, and (i) of the previous paragraph, we get $A \wedge B,\left(A^{\prime}\right)^{*} \vdash \perp$. From (ii) of the previous paragraph, $B^{\prime} \vdash B$, which holds by the way we have defined the set $\mathbb{A} \mathbb{C}(B)$, and $\neg(A \wedge B), A, B \vdash \perp$, which holds by logic, we get $\neg(A \wedge B), \neg\left(\left(A^{\prime}\right)^{*}\right), B^{\prime} \vdash \perp$. As noted in the previous paragraph, $\neg(A \wedge B) \cong \neg\left(A^{\prime} \wedge B^{\prime}\right)$; hence $\left\{\left(A^{\prime}\right)^{*}, B^{\prime}\right\}$ is completely and immediately less g-complex than $\neg(A \wedge B)$.

The third multiset $\left\{A \wedge B, \mathbf{B}^{*}[\mathbf{A}]\right\}$ can be analysed analogously to the previous one.

Lemma 5.2. (Soundness) For any $C, M$ and $A$, if there is a complete and immediate formal explanation of $\boldsymbol{A}$ from $\boldsymbol{M}$ under the robust condition $\boldsymbol{C}$ according to Definition 4.7, then there is an immediate and complete formal grounding 
relation between $M$ and $A$ under the robust condition $C$ according to Definition 3.7.

Proof. We analyze each rule belonging to Figure 3 showing that, for any $C, M$ and $A$, if $\mathbf{C}, \mathbf{M}$ and $\mathbf{A}$ are linked by one of these rules, then $\mathrm{C}, M$ and $A$ satisfy the three conditions in Definition 3.7.

Let $M=\{D, E\}$ and suppose that

\section{$\mathbf{D}, \mathbf{E}$ \\ $\widetilde{D \wedge E}$}

by means of the $\wedge G$ rule. So, by the form of the rule, $A \in \mathbb{A} \mathbb{C}(D \wedge E)$. Let $A$ $=(D \wedge E)^{\prime} \in \mathbb{A} \mathbb{C}(D \wedge E)$. We must prove that

(a) $D, E \vdash(D \wedge E)^{\prime}$

(b) $\neg D, \neg E \vdash \neg(D \wedge E)^{\prime}$

(c) $\{D, E\}$ is completely and immediately less g-complex than $(D \wedge E)^{\prime}$ according to Definition 3.6.

By the way we have defined the objects belonging to the set $\mathbb{A} \mathbb{C}(D \wedge E)$ we have that: $D \wedge E \vdash(D \wedge E)^{\prime}$, but also $\neg(D \wedge E) \vdash \neg(D \wedge E)^{\prime}$. From this and logic, we get conditions (a) and (b). Since $(D \wedge E)^{\prime} \cong D \wedge E,\{D, E\}$ is completely and immediately less g-complex than $(D \wedge E)^{\prime}$ and condition (c) is satisfied.

Let $M=\{D, E\}$ and suppose that

\section{$\mathbf{D}, \mathbf{E}$ \\ $\widehat{\mathbf{D} \vee \mathbf{E}}$}

by means of the $\vee G_{1}$ rule. So, by the form of the rule, $A \in \mathbb{A} \mathbb{C}(D \vee E)$. Let $A$ $=(D \vee E)^{\prime} \in \mathbb{A} \mathbb{C}(D \vee E)$. We must prove that

(d) $D, E \vdash(D \vee E)^{\prime}$

(e) $\neg D, \neg E \vdash \neg(D \vee E)^{\prime}$

(f) $\{D, E\}$ is completely and immediately less g-complex than $(D \vee E)^{\prime}$ according to Definition 3.6.

We establish (d)-(f) in the same way we have established the previous (a)-(c).

Let $M=\{D\}, C=E^{*}$ and suppose that

$$
\frac{\left[\mathbf{E}^{*}\right] \mathbf{D}}{\mathbf{D} \vee \mathbf{E}}
$$

by means of the $\vee G_{2}$ rule. (The analysis of the rule $\vee G_{3}$ is analogous.) So $A \in$ $\mathbb{A} \mathbb{C}(D \vee E)$ by the form of the rule. Let $A=(D \vee E)^{\prime} \in \mathbb{A} \mathbb{C}(D \vee E)$. We must prove that 
(g) $D \vdash(D \vee E)^{\prime}$

(h) $\neg D, E^{*} \vdash \neg(D \vee E)^{\prime}$

(i) $\left\{D, E^{*}\right\}$ is completely and immediately less g-complex than $(D \vee E)^{\prime}$ according to Definition 3.6.

By the way we have defined the objects belonging to the set $\mathbb{A} \mathbb{C}(D \vee E)$, we have that $D \vee E \vdash(D \vee E)^{\prime}$, but also $\neg(D \vee E) \vdash \neg(D \vee E)^{\prime}$. From this and the notion of converse of a formula, we get conditions (g) and (h). Since $(D \vee E)^{\prime} \cong D \vee E$, then $\left\{D, E^{*}\right\}$ is completely and immediately less g-complex than $(D \vee E)^{\prime}$ and condition (i) is satisfied.

Let $M=\{F, H\}, C=I$ and suppose that

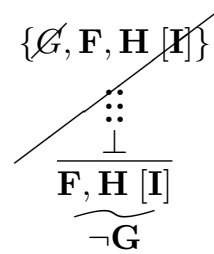

by means of the $\neg G$ rule. So, by the form of the rule, $A \in \mathbb{A} \mathbb{C}(\neg G)$. Let $A=\neg G^{\prime}$ $\in \mathbb{A} \mathbb{C}(\neg G)$. We must prove that, if $\{G, \mathbf{F}, \mathbf{H}[\mathbf{I}]\}$ is completely and immediately contradictory, then:

(j) $F, H \vdash \neg G^{\prime}$

(k) $\neg F, \neg H, I \vdash \neg \neg G^{\prime}$

(l) $\{F, H, I\}$ is completely and immediately less g-complex than $\neg G^{\prime}$ according to Definition 3.6.

Suppose that $\{G, \mathbf{F}, \mathbf{H}[\mathbf{I}]\}$ is completely and immediately contradictory. Then, by Lemma 5.1 , it follows that: (i) $G, \mathbf{F}, \mathbf{H} \vdash \perp$, (ii) $\neg G, \neg \mathbf{F}, \neg \mathbf{H}, \mathbf{I} \vdash \perp$ and (iii) $\neg G$ is completely and immediately more g-complex than $\{\mathbf{F}, \mathbf{H}, \mathbf{I}\}$. From (i)-(iii) and the way $\neg \mathbf{A}$ is defined, (j)-(l) immediately follow.

Lemma 5.3. (Completeness) For any $C, M$ and $A$, if there is an immediate and complete formal grounding relation between $A$ and $M$ under the robust condition $C$ according to Definition 3.7, then there is a complete and immediate formal explanation of $A$ from $M$ under the robust condition $C$ according to Definition 4.7.

Proof. The general strategy to prove this Lemma is as follows. First, we carefully list those multisets $N$ and formulas $D$ such that $N$ is completely and immediately less g-complex than $D$ according to Definition 3.6. Then, amongst these $N$ and $D$, we identify the pairs that stand in the positive, as well as in the negative, derivability relation. Those $N$ and $D$ such that, not only $N$ is completely and immediately less g-complex than $D$, but also $N$ and $D$ stand 
in positive and negative derivability relation, constitute cases of complete and immediate formal grounding; we thus check whether these cases can also be counted as cases of formal explanation, i.e. whether those $N$ and $D$ can constitute the premisses and the conclusion, respectively, of one of our grounding rules.

We proceed by reasoning on the g-complexity of $A$. If the g-complexity of $A$ is 0 , and thus $A$ is either an atom $p$ or the negation of an atom $p$, then there is nothing that is completely and immediately less g-complex than $A$ and hence nothing that stands in a complete and immediate formal grounding relation with $A$. If the g-complexity of $A$ is $>0$, then we distinguish two cases (I) $A \cong$ $\neg \neg B$ and (II) $A \cong(B \circ D)$. Let us first analyze (I).

(I) If $A \cong \neg \neg B$, then we have the following two sub-cases: (Ia) $A=(\neg \neg E)$ for some $E \in \mathbb{A} \mathbb{C}(B)$ (Ib) $A=(\neg \neg E)^{*}$ for some $E \in \mathbb{A} \mathbb{C}(B)$. In these cases, $B$ and $B^{*}$ are both completely and immediately less g-complex than $A$. But while $B$ enjoys a positive and negative derivability relation with $A$ in (Ia), $B^{*}$ enjoys a positive and negative derivability relation with $A$ in (Ib). Thus $B$ is a complete and immediate formal ground of $A$ in (Ia), while $B^{*}$ is a complete and immediate formal ground of $A$ in (Ib). A quick inspection of the rule $\neg G$ shows that such a rule ensures a complete and immediate formal explanation relation in the two aforementioned cases.

Let us now move to (II) where $A \cong(B \circ D)$. As before, let us first distinguish the following two sub-cases. (IIa) $A=E$, for some $E \in \mathbb{A} \mathbb{C}(B \circ D)$, (IIb) $A$ $=\neg E$, for some $E \in \mathbb{A} \mathbb{C}(B \circ D)$. For each sub-case, let us make some further distinctions:

$$
\begin{array}{ll}
\text { (IIa1) } A=E \text {, for some } E \in \mathbb{A} \mathbb{C}(B \wedge D) & \text { (IIb1) } A=\neg E \text {, for some } E \in \mathbb{A} \mathbb{C}(B \wedge D) \\
\text { (IIa2) } A=E \text {, for some } E \in \mathbb{A} \mathbb{C}(B \vee D) & \text { (IIb2) } A=\neg E \text {, for some } E \in \mathbb{A} \mathbb{C}(B \vee D)
\end{array}
$$

The following four multi-sets $\{B, D\},\left\{B, D^{*}\right\},\left\{B^{*}, D\right\},\left\{B^{*}, D^{*}\right\}$ are all completely and immediately less complex than $A$, in each form - from (IIa1) to (IIb2) - that $A$ might have. But when it comes to positive and negative derivability, a more subtle analysis is required. The combinations that can be obtained are the following:

$\odot\{B, D\}$ stands in a relation of positive and negative derivability with $A$, when $A$ is of the form (IIa1) and (IIa2);

$\ominus\left[D^{*}\right]\{B\}$ and $\left[B^{*}\right]\{D\}$ stand in a relation of positive and negative derivability with $A$, when $A$ is of the form (IIa2).

$\otimes[B]\left\{D^{*}\right\}$ and $[D]\left\{B^{*}\right\}$ stand in a relation of positive and negative derivability with $A$, when $A$ is of the form (IIb1).

$\oslash\left\{B^{*}, D^{*}\right\}$ stands in a relation of positive and negative derivability with $A$, when $A$ is of the form (IIb1), (IIb2). 
From $\odot$ and our previous classification in terms of complexity, it immediately follows that $\{B, D\}$ is the complete and immediate formal ground of $A$, when $A$ is of the form (IIa1), (IIa2). On inspection, the rule $\wedge G$ ensures a complete and immediate formal explanation relation between $\{B, D\}$ and $A$ when $A$ is of the form (IIa1); on inspection, the rule $\vee G_{1}$ ensures a complete and immediate formal explanation relation between $\{B, D\}$ and $A$ when $A$ is of the form (IIa2). Thus, each case of formal grounding is also a case of formal explanation.

From $\ominus$ and our previous classification in terms of complexity, it immediately follows that, when $A$ is of the form (IIa2), both $\{B\}$ under the robust condition $D^{*}$, and $\{D\}$ under the robust condition $B^{*}$, are the complete and immediate formal grounds of $A$. On inspection, the rule $\vee G_{2}$ and $\vee G_{3}$ ensure a complete and immediate formal explanation relation in these grounding cases.

From $\otimes$ and our previous classification in terms of complexity, it immediately follows that, when $A$ is of the form (IIb1), both $\left\{B^{*}\right\}$ under the robust condition $D$, and $\left\{D^{*}\right\}$ under the robust condition $B$, are the complete and immediate formal grounds of $A$. On inspection, the rule $\neg G$ ensures a complete and immediate formal explanation relation in both these grounding cases.

From $\oslash$ and our previous classification in terms of complexity, it immediately follows that $\left\{B^{*}, D^{*}\right\}$ is the complete and immediate formal ground of $A$, when $A$ is of the form (IIb1), (IIb2). On inspection, the rule $\neg G$ ensures a complete and immediate formal explanation relation in both these cases. Thus, each case of formal grounding is also a case of formal explanation.

Theorem 5.4. (Soundness and Completeness) The relation of complete and immediate formal explanation of Definition 4.7 is sound and complete with respect to the notion of complete and immediate formal grounding of Definition 3. $\%$.

Proof. It follows from Lemmas 5.2 and 5.3.

\section{Some remarks on the calculus $\mathcal{N G \mathcal { D }}$}

We use this section to emphasize certain important features of the calculus $\mathcal{N G D}$

First of all let us note that in the calculus $\mathcal{N G D}$ one can construct derivations as well as formal explanations. The possibility of constructing these two types of proofs in a single formal framework allows a deep understanding of the comparison between them. One of the most striking differences between derivations and formal explanations is that what in a formal explanation amounts to a single grounding step may, in a derivation, involve several inferential steps. A paradigmatic example is the following: while the derivation from the premises $A \wedge B, C$ to $(A \wedge C) \wedge B$ requires several inferential steps, the grounding step from $A \wedge B, C$ to $(A \wedge C) \wedge B$ is just one. This difference seems to correspond to the different conception of proofs that is behind derivations and formal explanations, respectively. While the former involves an epistemic conception of proofs, 
and hence each step of a proof may be viewed as a basic inferential passage of a valid reasoning, the latter concerns an etiological conception of proofs and thus each step of a proof corresponds to a different level of an ontological hierarchy.

Let us now turn to the second interesting feature of the calculus $\mathcal{N G D}$, which has already been partially mentioned in Section 2. In order to illustrate it, let us consider pairs of truths which are logically equivalent; as long as the truths belonging to each of these pairs also have the same g-complexity, then, according to the calculus $\mathcal{N G \mathcal { D }}$, they have the same complete and immediate formal grounds. This characteristic is quite central and has been neglected by all the logics of grounding introduced so far, with the exception of the logic built in [4]; it thus merits further discussion. First of all let us divide those pairs of truths that are equivalent but also have the same g-complexity into two sets: in one set there are those truths that share the same connectives, and in the other set there are those truths that have different connectives. Consider first this latter set, and take as exemple the truths $p \wedge q$ and $\neg(\neg p \vee \neg q)$. According to the De Morgan's law, $p \wedge q \equiv \neg(\neg p \vee \neg q)$. Moreover the g-complexity of $\neg(\neg p \vee \neg q)$ is 1 as is the g-complexity of $p \wedge q$. According to the rule $\wedge G$, the complete and immediate formal explanans of $p \wedge q$ are $\{p, q\}$; according to the rule $\neg G$, the complete and immediate formal explanans of $\neg(\neg p \vee \neg q)$ are $\left\{(\neg p)^{*},(\neg q)^{*}\right\}$, which is to say $\{p, q\}$. Thus $p \wedge q$ and $\neg(\neg p \vee \neg q)$ share the same complete and immediate formal explanans. This seems a reasonable and interesting result.

Let us now consider those pairs of truths that are equivalent, have the same g-complexity and also the same (occurrences of) connectives: these truths are those that have been previously defined as $a-c$ equiv, namely they are equivalent by applications of associativity and commutativity of conjunction and disjunction. In the calculus $\mathcal{N G \mathcal { D }}$ these truths have the same grounds. In $[14,13]$, the importance of building a logic where these truths have the same complete and immediate formal explanans has already been stressed. Note however that in a calculus where truths that are equivalent by applications of associativity and commutativity of conjunction and disjunction have the same grounds, grounds are no longer strictly speaking unique, as Bolzano conjectured (see [3, vol. IV, p. $266]$ ). In the calculus $\mathcal{N G \mathcal { D }}$ we have used the special notation of capital letters in bold character to refer to a-c equivalence classes of formulas: this notation has allowed us to have uniqueness in the grounding rules. In other terms, in our logic of grounding, grounds are unique up to a-c equivalence. The uniqueness requirement is not only close to Bolzano's intuitions (and to a conception of grounding as the objective explanation amongst truths), but also it allows us to have a calculus which is elegant and simple.

\section{Conclusions}

In this paper we have introduced a new logic for the notion of complete and immediate formal grounding; this logic has the form of the natural deduction calculus $\mathcal{N G \mathcal { D }}$ which involves both the relation of complete and immediate for- 
mal explanation and the connective of complete and immediate because. We think that $\mathcal{N G D}$ represents the basis for several and interesting lines of research. First of all, it would be important to extend the calculus $\mathcal{N G D}$ with grounding rules for implication as well as classical quantifiers: indeed we believe that the framework outlined above could provide an original and stimulating treatment

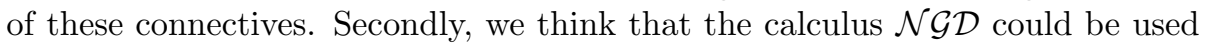
as a starting point for the study of the notions of complete and mediate, partial and immediate and partial and mediate grounding; by introducing new symbols and adequately adapting the existing ones, we could get an uniform formal analysis of all these notions. Finally we believe that a formal study of grounding can be considered complete only if an adequate semantics is provided. This is an important topic for future research.

Acknoweldgments I wish to thank Brian Hill for having corrected the English of the manuscript but also for several precious comments and suggestions. I would also like to thank the anonymous referees for their deep, clarifying and smart remarks.

\section{References}

[1] Aristotle. Posterior Analytics. 2nd edition. Oxford University Press, Oxford, 1994.

[2] R. Bliss and K. Trogdon. Metaphysical grounding. In E. N. Zalta, editor, The Stanford Encyclopedia of Philosophy, pages 1-42. 2016.

[3] B. Bolzano. Theory of Science. Oxford University Press, Oxford, 2015.

[4] F. Correia. Grounding and truth-functions. Logique et Analyse, 53(211):251-79, 2010.

[5] F. Correia. Logical grounds. Review of Symbolic Logic, 7(1):31-59, 2014.

[6] F. Correia. On the logic of factual equivalence. Review of Symbolic Logic, 9(1):103-122, 2016.

[7] F. Correia and B. Schnieder. Grounding: an opinionated introduction. In F. Correia and B. Schnieder, editors, Metaphysical grounding, pages 1-36. Cambridge University Press, Cambridge, 2012.

[8] K. Fine. Some puzzles of ground. Notre Dame Journal of Formal Logic, 51(1):97-118, 2010.

[9] K. Fine. Guide to ground. In F. Correia and B. Schnieder, editors, Metaphysical grounding, pages 37-80. Cambridge University Press, Cambridge, 2012 . 
[10] K. Fine. The pure logic of ground. Review of Symbolic Logic, 25(1):1-25, 2012.

[11] S. Krämer and S. Roski. A note on the logic of worldly ground. Thought, 4:59-68, 2015.

[12] F. Poggiolesi. Getzen Calculi for Modal Propositional Logic. Springer, 2010.

[13] F Poggiolesi. A critical overview of the most recent logics of grounding. In F. Boccuni and A. Sereni, editors, Objectivity, Realism and Proof, pages 1-18. Boston Studies in the Philosophy and History of Science, 2016.

[14] F. Poggiolesi. On defining the notion of complete and immediate formal grounding. Synthese, pages 1-24, 2016.

[15] A. Rumberg. Bolzano's theory of grounding against the background of normal proofs. Review of Symbolic Logic, 6(3):424-459, 2013.

[16] J. Schaffer. On what grounds what. Metametaphysics, pages 347-383, 2009.

[17] B. Schnieder. A puzzle about 'Because'. Logique et Analyse, 53(4):317-343, 2010 .

[18] B. Schnieder. A logic for 'Because'. The Review of Symbolic Logic, 4(03):445-465, 2011.

[19] J. Sebestik. Logique et mathematique chez Bernard Bolzano. J. Vrin, 1992.

[20] A. Tatzel. Bolzano's theory of ground and consequence. Notre Dame Journal of Formal Logic, 43(1):1-25, 2002.

[21] A. S. Troelstra and H. Schwichtenberg. Basic Proof Theory. Cambridge University Press, 1996.

[22] D. van Dalen. Logic and Structure. Springer-Verlag, Dordrecht, 1991.

[23] H. Wansing. Sequent calculi for normal modal propositional logics. Journal of Logic and Computation, 4:125-142., 1994. 